\title{
Global capacity announcement of electrical distribution systems: A pragmatic approach
}

\author{
Bertrand Cornélusse $^{\mathrm{a}}$, David Vangulick ${ }^{\mathrm{b}}$, Mevludin Glavic ${ }^{\mathrm{a}}$, Damien Ernst ${ }^{\mathrm{a}}$ \\ ${ }^{a}$ Dept. of Electrical Engineering and Computer Science, University of Liège, Sart Tilman B28, B-4000 Liège, Belgium \\ (e-mail: bertrand.cornelusse@ulg.ac.be; fax: + 324366 2984). \\ ${ }^{b}$ ORES, Louvain-la-Neuve, Belgium.
}

\begin{abstract}
We propose a pragmatic procedure to facilitate the connection process of Distributed Generation (DG) with reference to the European regulatory framework where Distribution System Operators (DSOs) are, except in specific cases, not allowed to own their generation. The procedure is termed Global Capacity ANnouncement (GCAN) and is intended to compute the estimates of maximum generation connection amount at appropriate substations in a distribution system, to help generation connection decisions. The pragmatism of the proposed procedure stems from its reliance on the tools that are routinely used in distribution systems planning and operation, and their use such that the possibilities of network sterilization are avoided. The tools involved include: long-term load forecasting, long-term planning of network extension/reinforcement, network reconfiguration, and power flow. Network sterilizing substations are identified through repeated power flow computations. The proposed procedure is supported by results using an artificially created 5-bus test system, the IEEE 33-bus test system, and a part of real-life distribution system of ORES (a Belgian DSO serving a large portion of the Walloon region in Belgium).
\end{abstract}

Keywords: Distributed generation, system capacity announcement, connection decisions, repeated power flow, reconfiguration, planning, distribution system operator.

\section{Introduction}

Legislative and regulatory frameworks, in many countries around the World, promote connections of renewable generation in distribution systems [1]. A well-designed generation connection process is the key prerequisite to increase the penetration level of this generation and meet legislative or regulatory targets. However the penetration level of renewable generation is often hampered by a lack of coordination among generation connection developers and DSO in planning future connections.

Existing, mostly research, approaches for DG siting and sizing are reviewed in [2]. While many approaches exist they often, with few exceptions, neglect practical aspects of the problem and possible obstacles for their implementation. In practice, a still dominant approach to generation connections is firstcome first-serve based on computations of connections for substations individually and hence potentially leading to the system sterilization. The sterilizing substations, within a system, are those in which locating inappropriate generation amount prevents larger connections in other substations and thus considerably reducing the system hosting capacity [3].

Legislative and regulatory frameworks vary from one country to another and an important aspect related to the connection process is the ownership of DGs. Within the European regulatory framework [4-7] DSOs are not allowed to own generation plants, except for a small number of legacy plants [8] or to manage system losses as in the Belgian Walloon region [9]. This considerably impacts planning activities of DSOs since the lack of coordination between network and generation planning can lead to inefficiencies in electricity supply infrastructure.
This problem was considered in several previous works. In [8] the need for schemes and practices to provide incentives for DG deployment for the benefit of the network were identified considering different regulations for DSO ownership of DG. This work specifically criticized European regulatory framework and points out difficulties brought by the fact DSOs are not allowed to own generation. Through the use of optimal power flow to evaluate a distribution network capacity, the authors of [10] clearly identify the threat of network sterilization, by siting generation at inappropriate substations. One of the main points raised in [10] is: "Whereas the technical problems arising from distribution-level connections may be mitigated for individual connections, the anticipated connection volumes imply a potential risk of conflict between connections, in that inappropriately sized or located plant could constrain further development of the network and consequently threaten the achievement of renewable energy targets". Recognizing that the system sterilization stems from capacity allocation to the substations whose voltage and/or short-circuit levels are most sensitive to power injections, reference [3] proposed a sensitivity based linear programming method to determine the optimal generation siting and sizing. Sensitivity based approach was also considered in [11].

This work, along the line of [1], argues that a more proactive approach of DSOs is needed in order to facilitate higher levels of renewable generation penetration. We suggest that such an approach should include the study of the system in an effort to approximately determine its hosting capacity in advance. The results of this study should be made available publicly through 
a website [12], the system locational maps [13], or upon request from connection developers.

Some DSOs already have in place a sort of procedure to approximately compute generation connection amount and make the information public. These procedures, as well as the way their results are published, differ depending on the confidentiality considerations that apply to the DSO. Most of UK DSOs provide the long term development statement [13]. This statement usually includes network data over the planning horizon (usually five years) and it is a generation connection potential developer who takes the data, chooses an appropriate tool and computes available capacity at substations of his interest. Several DSOs in Canada practice approach in which the DSO computes and publishes results of capacity assessment but also provides, DSO hosted, station and feeder capacity calculator that can be used by the developer [12].

The GCAN procedure proposed in this work, similarly to approaches of [12, 13], is intended to approximately compute the generation connection amount in all or pre-specified system substations, but also accounts for the DSO investment plan and some other targets such as renewable penetration and losses minimization. Its purpose is to attract and encourage developers for generation connection projects. We believe that defining well the complete GCAN procedure helps in making the whole connection process transparent. This procedure is a result of research efforts undertaken within the framework of the GREDOR project [14] and under scrutiny of Belgian Walloon region regulator $\mathrm{CWaPE}$ to be an integral part of connection process in the region.

The core of the proposed procedure is an efficient use of power flow in repeated way such that the system sterilization is avoided.

This work is organized as follows. The GCAN procedure, with a detailed presentation of the proposed repeated power flow, is presented in Section 2. Section 3 presents three systems used for illustration of the procedure, while section 4 details results obtained with these three systems, together with the validation through comparison with an optimal power flow solution and discussions on possible improvements. Section 5 concludes.

\section{GCAN computation}

The procedure is developed with the following premises:

1. it should be in line with the network long-term development plans,

2. it should take into account target levels of DG penetration and any other specific targets (e.g., target level of system losses with respect to predicted load) and these targets are set by legislative and regulatory bodies (not by DSO),

3. it should rely on the tools that are routinely used by DSOs,

4. DSO operates its system with optimal network configuration determined with the aim of active power losses minimization.

Item 1 above implies that a DSO performs long-term load forecast and network expansion/reinforcement plan over a usual

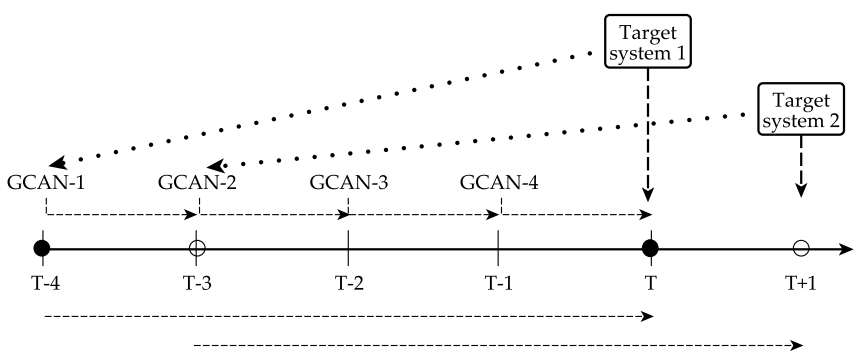

Figure 1: Idea of dynamic GCAN computation

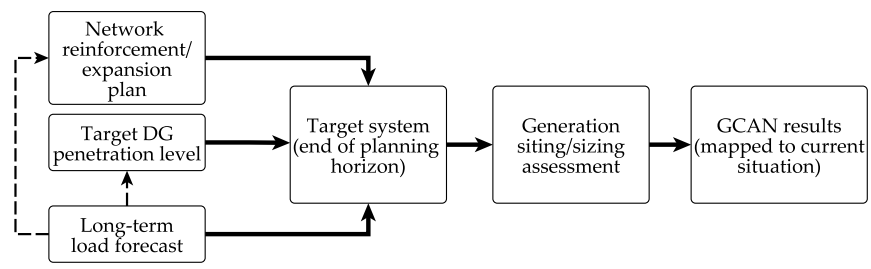

Figure 2: Dynamic GCAN computation: inputs and outputs

planning horizon using existing tools. Taking into account the targets (item 2) refers to the system situation at the end of the planning horizon where the targets are simply computed as a percentage of the system load, and taking into account the generation units that are already contracted but not yet connected, in commissioning, or not yet at full power. Item 3 ensures simplicity of the overall procedure by relying on an existing power flow tool (used as repeated power flow computation) to identify sterilizing substations and compute the maximum generation amount in the remaining ones. Item 4 implies running optimal network reconfiguration for active power losses reduction [15] since it has potential to increase system capacity [16].

The idea of GCAN is illustrated in Fig. 1 while inputs and outputs needed for the computations are shown in Fig. 2 The main idea is to define the target system corresponding to the end of the planning horizon. The target system is defined in terms of:

- long-term prediction of load growth,

- target network as the result of network reinforcement and expansion plan,

- target penetration level of distributed generation (ratio of generation and load level) that could be based on regulatory or environmental considerations,

- generation units which are already contracted but not yet connected, in commissioning, or not yet at full power,

- target system active power losses as percentage of the total net injections, and

- possibly complemented with other targets (power quality requirements, etc.). 


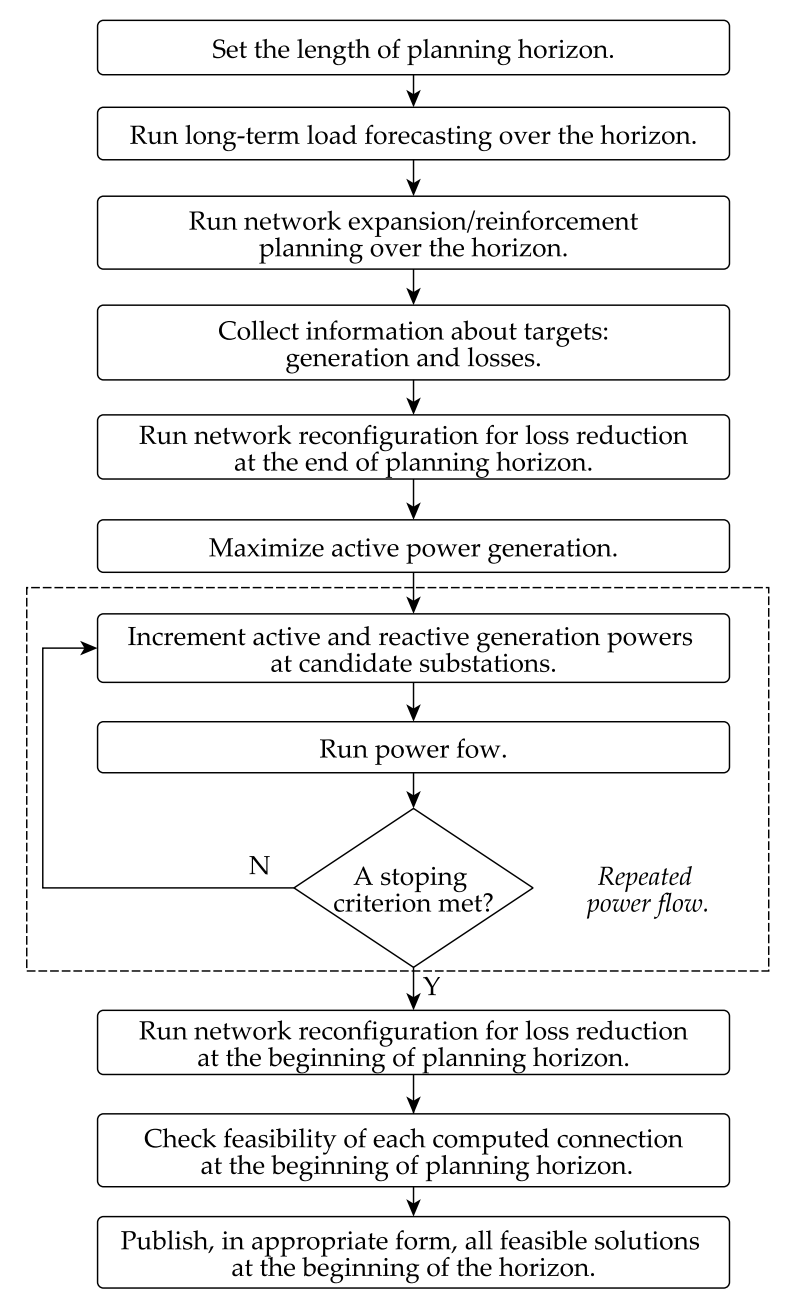

Figure 3: GCAN procedure

Based on the above considerations the GCAN procedure is shown in Fig 3 . Four computational tools are needed to complete the procedure: long-term load forecast, network expansion/reinforcement planning, optimal reconfigurations, and repeated power flow. We do not suggest any specific tool for load forecast and network expansion/reinforcement (existing tools used routinely by a DSO for these purposes should be used), but we suggest specific implementations of network reconfiguration and repeated power flow, built around an existing power flow tool.

The results of GCAN are computed regularly (at each step of planning horizon) but also updated immediately after a new significantly large connection is realized on the system. The computation is conducted in a rolling horizon manner. In this way the GCAN routinely updates or revises the computations taking into consideration more reliable and recent data as they become available. Consequently, at the next planning step GCAN takes into account new network planning results, any network expansion/reinforcement and generation connections realized during the first period of the horizon, while the horizon itself if shifted by one planning period.

\subsection{Optimal network reconfiguration}

Many algorithms were proposed for optimal network reconfiguration [15, 16]. Through extensive testing of different algorithms we found that the heuristic one of [15] is particularly useful based on its simplicity and ability to attain the solution comparable with more sophisticated algorithms. It relies on a power flow tool and thus considers non-linearity of the system and avoids usual approximations involved in many reconfiguration algorithms. The algorithm starts with the definition of the list of all maneuverable lines and the network configuration corresponding to all lines closed. The algorithm proceeds with opening lines one by one, solving power flow for each line opened, and takes the decision to open the line that has resulted in the smallest increase in the active power loses. The procedure is stopped once all maneuverable lines are removed from the created list [15]. In this work we slightly modified the algorithm of [15] by considering all lines as maneuverable and introducing a radiality check of the corresponding graph as stopping criterion.

\subsection{Repeated power flow}

Repeated power flow (RPF) is a simple extension of conventional power flow. It solves the parameterized conventional power flow equations,

$$
f(x, p)=0
$$

where $x$ is the vector of state variables (bus voltage magnitudes and angles) and $p$ a vector of parameters (usually load power withdrawals, or as in this paper generation power injections). Equations (11) are solved for each incremental change of the parameter vector $p$ and the entries of this vector define a specific direction of the changes.

This power flow extension is in practical use to solve some power system problems, most notably to compute available transfer capability and the system loadability with respect to voltage instability [17]. RPF has also been mentioned in [11] as a tool to compute generation connections in distribution systems and used to validate the proposal of [11]. This reference considered RPF to compute generation connection amounts in a sequential manner, without resolving the problem of order in the sequence. We demonstrate drawbacks of this approach in a latter section of this work and argue that the RPF should be used to compute generation connections simultaneously. The ease of implementation is the biggest advantage of RPF while computational burden is of concern and a care should be taken for efficient implementation of this method [17].

Similarly to the use of RPF for transfer capability and voltage stability related loadability computation, for an efficient implementation of power flow in repeated way a candidate substation list (CSL) and step increase in active power generation should be defined. Let the step increase in active power be defined as,

$$
\Delta P_{g_{i}}=\frac{d_{i}}{\sum_{j \in C S L} d_{j}}, i \in C S L
$$

Parameters $\Delta P_{g_{i}}$ should be set to reflect how individual substations are distant from the corresponding limit. We suggest the 
use of approximate distances. The distance for substation $i$ is derived from its complex power,

$$
\bar{S}_{i}^{*}=\bar{V}_{i}^{*} \sum_{j=1}^{n} \bar{Y}_{i, j} \bar{V}_{j}
$$

and apparent power computed as,

$$
S_{i}=V_{i}\left\|\sum_{j=1}^{n} \bar{Y}_{i, j} \bar{V}_{j}\right\|
$$

The approximate distance for substation $i$ is then computed by taking derivative of the apparent power (eq44) with respect to the voltage magnitude of the substation and multiplying it with voltage range (difference between present and maximum allowed values),

$$
d_{i}=\left(2 V_{i}\left\|Y_{i, i}\right\|+\sum_{j=1, j \neq i}^{n} V_{j}\left\|Y_{i, j}\right\|\right)\left(V_{i, l i m}-V_{i}\right), i \in C S L
$$

where $\left\|Y_{i, i}\right\|$ is the absolute value of the admittance matrix entry $(i, i)$. Admittance matrix entries are easily available with every standard power flow program. The distance (4) is an approximation since the modulus of the sum used in the above equations is always less or equal to the sum of the modula of individual components. This distance approximately computes the amount of MVA that could be added in a substation (bus) before the voltage magnitude at this substation reaches its limit (usually $V_{l i m}=1.05$ since the upper voltage limit is of concern when considering new generation connections).

Reactive power is changed according to the change of active power, using a predefined power factor. These parameters are re-computed at each RPF run. This way the procedure takes into account the robustness of individual substations and it is expected that preference in generation connection would be driven towards substations that are the least sensitive to generation injections [3].

The proposed RPF procedure takes into account the voltage magnitude, fault level, and thermal constraints of the elements of the system. If $S C L_{i}$ is the short-circuit level (in MVA) at the substation $i$, the corresponding fault level constraint for generation connection is defined for each substation $i \in C S L$ as

$$
\Delta P_{g_{i}} \leq 0.1 S C L_{i} \cos \left(\theta_{i}\right) .
$$

The procedure for RPF is summarized as follows:

1. Initialization:

- define the candidate substations list (CSL),

- compute the active power step increase for all CSL substations using (2),

- define the generation power factor for all CSL,

2. while stopping criteria are not met:

- run power flow

- update:
- if any voltage is outside the interval $[0.95,1.05]$ or $S C L$ is reached (say at substation $i$ ) then set $\Delta P g_{i}=0$, and further check if the computed $P g_{i}$ is below predefined threshold, if yes then set $P g_{i}=0, Q g_{i}=0$

- if a thermal constraint is violated then freeze generation at all substations downstream of limited line

- for the remaining substations

$$
\begin{aligned}
& * P g_{i} \leftarrow P g_{i}+\Delta P_{g_{i}}, i \in C S L, \\
& * Q g_{i} \leftarrow P g_{i} \tan \left(\theta_{i}\right), i \in C S L .
\end{aligned}
$$

The stopping criteria are:

1. the thermal limit of the connection transformer is reached,

2. the level of target active power losses is reached,

3. no further increase in active power of any candidate substation is possible without violating constraints (voltage, thermal, and fault level).

The procedure is illustrated in Fig. 4. It starts with an initial direction of generation increase. If a voltage limit is reached at a substation, the corresponding generation connection is handled as follows. If the computed generation amount is smaller or equal than a pre-defined small amount, then it is set to zero (generation is disconnected and the corresponding $\Delta P_{g_{i}}$ is set to zero); this could correspond to points 1 and 2 in Fig. 4 Then any of the following options could correspond to point 3 in Fig. 4 .

- the generation amount exceeds the SCL for a substation then the corresponding generation is fixed to the reached value and the corresponding $\Delta P_{g_{i}}$ is set to zero;

- the generation amount is greater than a pre-defined threshold and smaller than a corresponding SCL - then the generation is fixed to the reached value and excluded from further generation increase (the corresponding $\Delta P_{g_{i}}$ is set to zero);

- the thermal limit of a line is reached - then all generations downstream of the limited line are fixed to the reached value and excluded from further generation increase (corresponding $\Delta P_{g_{i}}$ are set to zero);

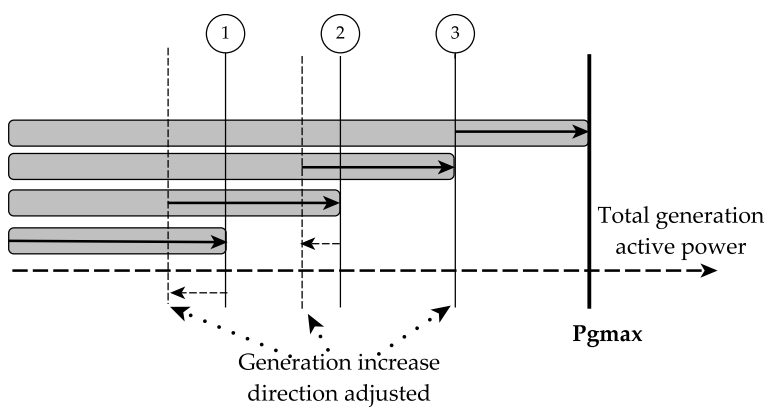

Figure 4: Illustration of RPF for simultaneous maximum generation increase The point $P_{\text {gmax }}$ corresponds to one of the three stopping criteria. 


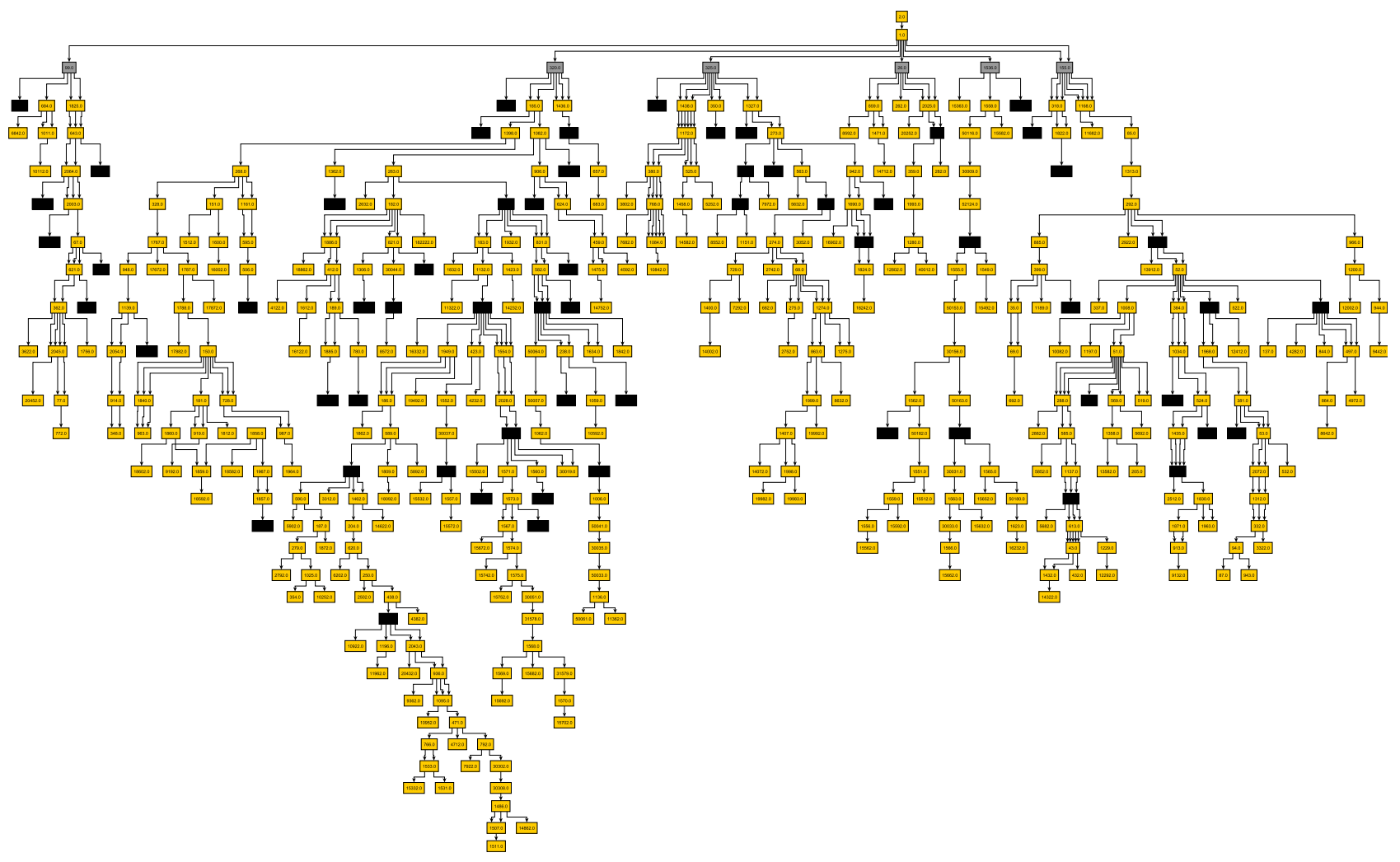

Figure 7: RL system: optimal configuration and network reinforcement plan.

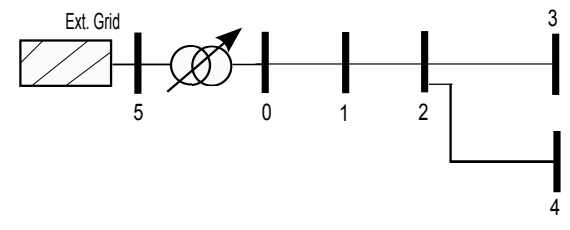

Figure 5: Artificial 5 bus test system

\section{Test systems}

Three systems are used to test the proposed approach: an artificial 5-bus (Fig 5), IEEE 33-bus test system (Fig 6) [16], and a real-life subsystem of ORES termed here as RL system (Fig 7).

The main characteristics of these systems are summarized in Table 1 presenting: nominal voltage $V$, number of buses (substations) $n_{b}$, number of lines $n_{l}$, number of generators $n_{g}$, total active power load power $P_{l}$, total reactive load power $Q_{l}$, and total generation active power $P_{g}$.

Table 1: Characteristics of test systems

\begin{tabular}{cccccccc}
\hline System & $\begin{array}{c}V \\
k V\end{array}$ & $n_{b}$ & $n_{l}$ & $n_{g}$ & $\begin{array}{c}P_{l} \\
M W\end{array}$ & $\begin{array}{c}Q_{l} \\
M V A r\end{array}$ & $\begin{array}{c}P_{g} \\
M W\end{array}$ \\
\hline 5-bus & 13.80 & 5 & 4 & 0 & 4.00 & 1.00 & 0 \\
\hline 33-bus & 12.66 & 33 & 37 & 0 & 3.72 & 2.30 & 0 \\
\hline RL & 10.00 & 424 & 579 & 6 & 10.34 & 3.41 & 12 \\
\hline
\end{tabular}

\section{Results}

The presented results illustrate the following:

- the Small 5-bus test system is used to show the capability of the proposed RPF to identify sterilizing substations,

- the IEEE 33-bus test system is used to highlight the importance of considering network reconfiguration in GCAN, and full elaboration of GCAN results.

- the RL system is used to demonstrate the capabilities of the proposed GCAN of dealing with real-life systems.

MATPOWER [18] is used for power flow computations in all simulations that produced results included in this work. This tool implements a so-called concentrated load model where the load is represented by lumped active and reactive powers. Generation powers, both active and reactive, at CSL substations are considered as negative loads.

\subsection{Results for 5-bus system}

In this section we illustrate the capability of RPF, with simultaneous generation increase, to identify all sterilizing buses and that the results are comparable with other, but more complicated, procedures considered in literature [10]. To this purpose RPF is run in two modified modes: individual (each substation is considered as candidate separately) and sequential (generation connections are computed sequentially for each substation assuming previously computed ones are connected). The results are given in Table 2. These results are produced starting 


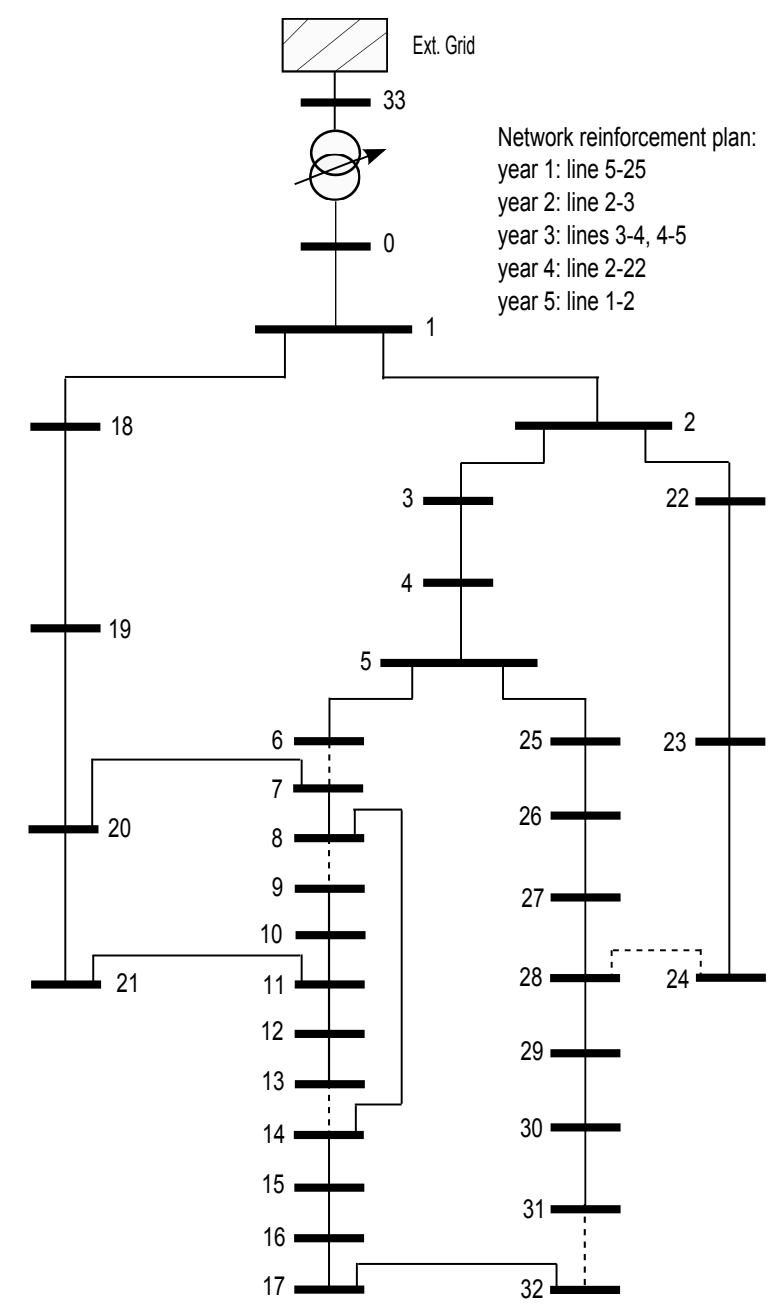

Figure 6: IEEE 33 bus test system: optimal configuration and network reinforcement plan.

from a normal operating point, considering three substations as candidate ones $(2,3$, and 4$)$ and a unity generation power factor in all substations.

RPF in individual mode can be considered as a proxy to optimal power flow used in [10]. The values shown for this mode in Table 2 cannot be connected together as discussed in [10] since system development is not taken into account and the order of connection has an important impact on real connections. In order to illustrate this, sequential RPF are run with three different orders of connection. If generations are connected either in substation 3 or 4 it considerably limits connections in the other

Table 2: Generation connections for the 5-bus system

\begin{tabular}{cccc}
\hline RPF & $P_{2}$ & $P_{3}$ & $P_{4}$ \\
& $M W$ & $M W$ & $M W$ \\
\hline Individual & 6.79 & 4.23 & 4.24 \\
\hline Sequential-234 & 6.79 & 0 & 0 \\
\hline Sequential-324 & 0.05 & 4.23 & 0.03 \\
\hline Sequential-423 & 0.09 & 0.07 & 4.24 \\
\hline Simultaneous & 6.79 & 0 (sterilizing) & 0 (sterilizing) \\
\hline
\end{tabular}

two substations and the total penetration of DG (4.31 MW if generation is connected first in substation 3 and 4.40 MW if generation is connected first in substation 4). If generation is connected first in substation 2 it leaves no room for any other connection in the other considered substations resulting in a total generation amount of $6.79 \mathrm{MW}$. Clearly, the best solution would be to publish a capacity of $6.79 \mathrm{MW}$ for substation 2 and zero for the two others (a similar conclusion was drawn in [10] using a realistic test system). However, in order to come up with an optimal order of connection, additional and tedious work on different order combinations is needed.

RPF with simultaneous generation increase is able to identify substations 3 and 4 as sterilizing in an automated way and avoids complications introduced by the combinatorial nature of finding an optimal order of connection. It gives as final solution the connection in substation 2 with an amount of 6.79 MW, as shown in Table 2 First, substation 3 is identified as sterilizing when the total generation reached the value of $6.22 \mathrm{MW}$ (with individual generations of $1.87 \mathrm{MW}, 2.02 \mathrm{MW}$, and 2.33 MW for substations 2, 3, and 4, respectively). Next, substation 4 is identified as sterilizing when, after disconnection of generation at substation 43 the total generation reached $6.45 \mathrm{MW}$ with individual connections as $3.53 \mathrm{MW}$ for substation 2 and $2.88 \mathrm{MW}$ for substation 4 . No pre-defined minimum value for generation connection was used in this case. A substation is declared as sterilizing if the corresponding voltage limit is reached.

\subsection{Results for IEEE 33-bus and RL systems}

All the results, for these two systems, are produced assuming the following conditions. DSOs are planning investments over a five years horizon (following the practice of UK DSOs to provide five year system development statement [13]). No specific toll is used for long-term load forecast and simply $3 \%$ of load growth per year is assumed. Peak loads are assumed $20 \%$ higher than base loads given in Table 1 while the minimum loads are set to be $40 \%$ of the peak, for all substations, No specific tool for network expansion/reinforcement is used but only network reinforcement is considered by running power flows for each year within the planning horizon and observing overloaded lines to be reinforced at that particular year. All substations, except primary and secondary sides of the transformer connecting the DSO system to the transmission network and those substations in which generation already exist at the beginning of the planning horizon, are considered as candidate for generation connection. The active power losses target is set to $3 \%$ of the connection transformer thermal capacity. Pre-defined minimum generation connection values are set to $0.5 \mathrm{MW}$ for the IEEE 33-bus and 0.2 MW for the RL system. In order to mimic different possible technological solutions for generation connection three different power factors of generators are considered: 0.95 (leading), 1, and 0.95 (lagging). In deciding which power factor to use for candidate substations it is assumed that the DSO has the knowledge of preferred technological solutions for all the substations, and all the results are obtained for minimum load conditions and setting the secondary voltage of the connection transformer, and the controllable ex- 
Table 3: Results of GCAN computation to publish for the first year

\begin{tabular}{cccc}
\hline Substation & $\begin{array}{c}\text { MW } \\
\text { end }\end{array}$ & $\cos \theta$ & $\begin{array}{c}\text { MW } \\
\text { individual }\end{array}$ \\
\hline 2 & 3.62 & 0.95 (lead) & 7.15 \\
\hline 3 & 2.72 & 1.00 & 4.71 \\
\hline 4 & 2.19 & 1.00 & 3.64 \\
\hline 18 & 2.84 & 1.00 & 11.04 \\
\hline 19 & 0.64 & $0.95($ lag) & 2.12 \\
\hline 20 & 0.79 & $0.95($ lag) & 1.82 \\
\hline 22 & 1.36 & 0.95 (lead) & 5.40 \\
\hline 23 & 0.74 & $0.95($ lead $)$ & 3.37 \\
\hline 28 & 0.74 & $0.95($ lead $)$ & 1.57 \\
\hline 29 & 0.72 & 1.00 & 1.46 \\
\hline
\end{tabular}

isting generators (if applicable), to their lower limit ( $0.95 \mathrm{pu})$ for the following reasons:

- both systems represent rural network and voltage raise is of primary concern, this implies the consideration of system conditions corresponding to minimum load,

- in order to allow bigger generation connections, voltage control devices should be set to the minimum voltages (in the IEEE 33-bus system only the tap changer of the connection transformer is considered as a voltage control device while in the RL system both the tap changer of the transformer and some of the existing generators are considered for voltage control).

With the results related to the IEEE 33-bus test system, we provide a detailed presentation of GCAN results. This is followed by a demonstration of the importance of computing generation connections for an optimally configured network. The optimal network configuration corresponding to the final year of the planning horizon together with the network reinforcement plan are shown in Fig 6 where dashed lines indicate opened branches. For all lines the thermal limit is set to 6.66 MVA, except for the connection transformer (26.64 MVA) and lines 1-2, 2-3, 2-22, 3-4, 4-5, and 5-25 (13.32 MVA).

The results of GCAN computations are summarized in Table 3 including the power factor set to each substation and connection powers computed as individual connections of each substation at the beginning of the horizon. As can be seen from this table all the connections computed for the end of the planning horizon are feasible as individual connections at the beginning of the horizon and the information for all substations should be published for the first year of the planning horizon.

Figure 8 shows the evolution of the total generation amount during RPF computations and indicates power flow runs in which six substations are identified as sterilizing. RPF is stopped by the active power losses criterion in the 24-th run.

The results of the impact of network configuration are given in Table 4 These results are produced under the same conditions as for results shown in Table 3, and they confirm the suggestion of [16] related to the potential of network reconfiguration to increase DG penetration. In this particular case the optimal network configuration gives $1.03 \mathrm{MW}$ more then in case

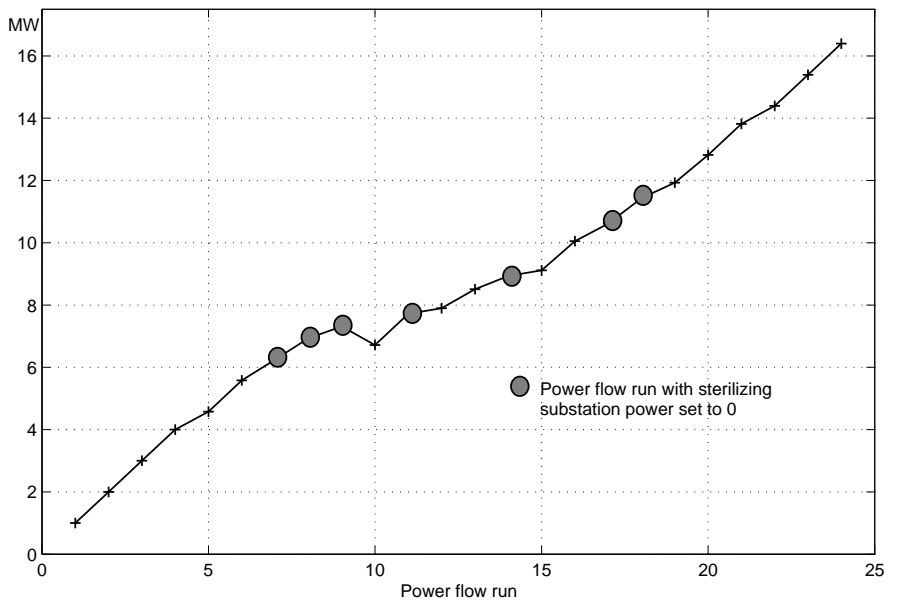

Figure 8: RPF runs

the system is operated in usual configuration. A small difference in the connection amounts is explained by the fact that the usual configuration losses are a little higher than with the optimal configuration without any generation connection (0.039 with respect to $0.03 \mathrm{MW}$ ) indicating that the usual configuration is close to optimal. At the next step in the planning horizon all the procedure is repeated by refreshing the data (taking into account network reinforcement in the first year, possible generation connections during the first year, etc.). Assuming the generator is connected at substation $22(1.36 \mathrm{MW})$ during the first year, the new network expansion/reinforcement reveals it cancels reinforcement of the lines 2-3 and 3-23. The time horizon is shifted for one step (assuming an additional 3\% of load increase in the last year). Computations for the second year are given in Fig 9 in grey color bars and compared to the first year values (shown in black bars). The results show that the modified network expansion/reinforcement plan impacts the result and in general slightly lower generation connections are obtained as compared to the first year. However, these connections together are still much above the penetration targets.

In the RL system, six DGs already exist (indicated with grey boxes in Fig 7) and all of them are wind generators. It is assumed this technology is preferred in the whole RL system and the power factor is set to 0.95 (leading) for all candidate substations. Since the existing generation of $12 \mathrm{MW}$ already exceeds the system load at the beginning of the planning horizon (10.34 MW), DG penetration target for this system is set $300 \%$ of the system load at the end of the planning horizon. The results of GCAN computation, for this system, are shown in Fig 7 where generation connections are indicated by black boxes and in Fig 10 where amounts, to be published in the first year, are

Table 4: Impact of network configuration

\begin{tabular}{ccc}
\hline Configuration & $\begin{array}{c}\text { Open } \\
\text { switches }\end{array}$ & $\begin{array}{c}P_{\text {total }} \\
\text { MW }\end{array}$ \\
\hline Usual & $7-20,8-14,11-21,17-32,24-28$ & 15.34 \\
\hline Optimal & $6-7,8-9,13-14,24-28,31-32$ & 16.37 \\
\hline
\end{tabular}




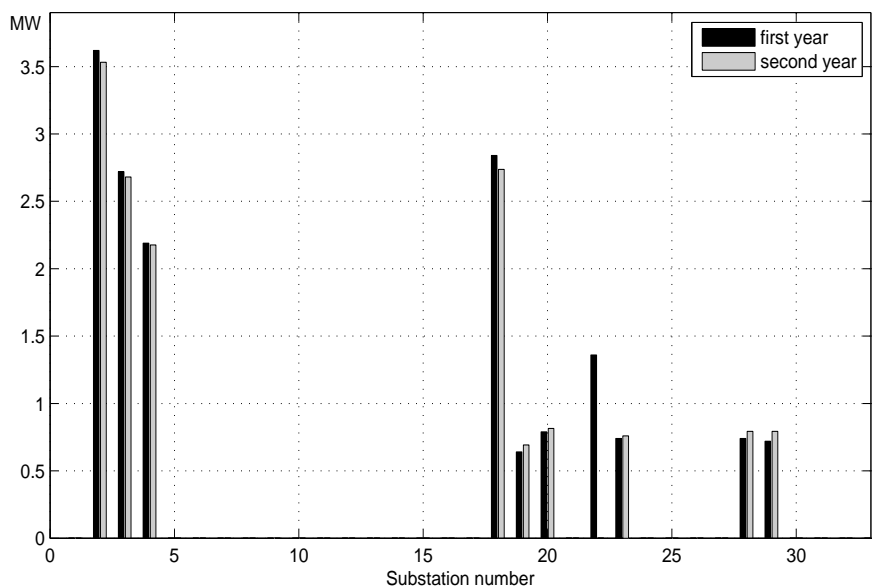

Figure 9: Generation connections for IEEE 33-bus system: first and second year of planning horizon

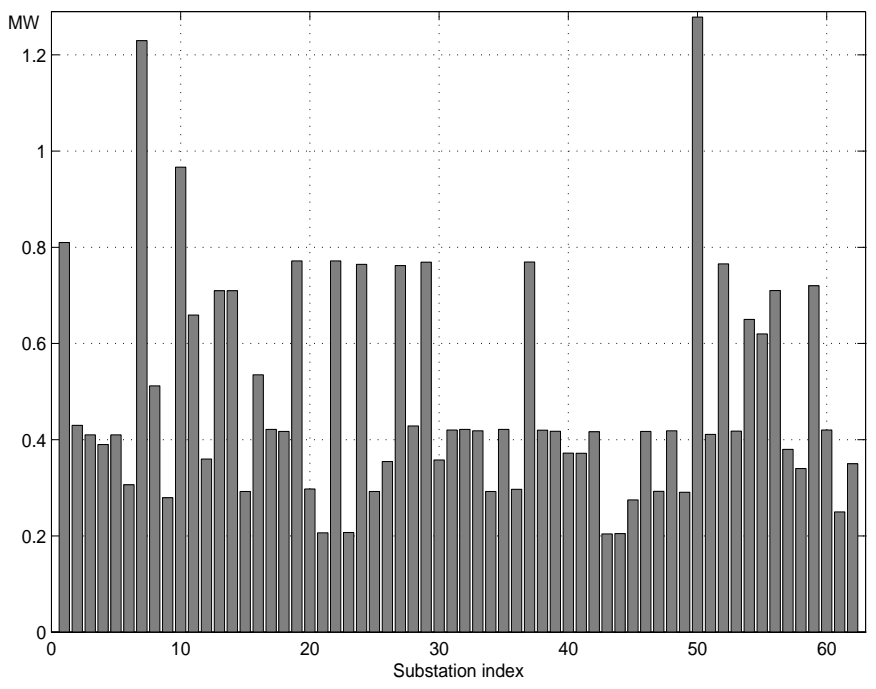

Figure 10: Generation connections for RL system: corresponds to the system one line diagram in Fig 7 (from left to right)

shown in a bar diagram corresponding to the map of Fig 7. Out of 424 substations 362 are found as sterilizing. The total generation is $30.28 \mathrm{MW}$.

\subsection{Validation of the proposed procedure}

The results obtained for the IEEE 33-bus test system are validated through comparison with a full non-linear Optimal Power Flow (OPF) problem. The latter problem is formulated as a Mixed Integer Non-Linear Program (MINLP), modeled in Python using Pyomo [19] and solved with BONMIN [20]. The details of the MINLP formulation are given in Appendix. Table 5 summarizes the comparison results.

As expected the OPF formulation gives more capacity and the error of RPF is about $3 \%$. However the simplicity of the proposed procedure and its relatively small error qualify it as a useful helper in the generation connection process.
Table 5: Comparison of RPF and OPF

\begin{tabular}{cccc}
\hline Substation & RPF (MW) & OPF (MW) & Difference (MW) \\
\hline 2 & 3.62 & 3.77 & 0.15 \\
\hline 3 & 2.72 & 2.97 & 0.25 \\
\hline 4 & 2.19 & 2.17 & -0.02 \\
\hline 5 & 0.00 & 1.01 & 1.01 \\
\hline 10 & 0.00 & 0.52 & 0.52 \\
\hline 18 & 2.84 & 2.90 & 0.06 \\
\hline 19 & 0.64 & 0.65 & 0.01 \\
\hline 20 & 0.79 & 0.87 & 0.08 \\
\hline 22 & 1.36 & 1.41 & 0.05 \\
\hline 23 & 0.74 & 0.54 & -0.20 \\
\hline 28 & 0.74 & 0.00 & -0.74 \\
\hline 29 & 0.72 & 0.00 & -0.72 \\
\hline Total & 16.37 & 16.8 & 0.43 \\
\hline
\end{tabular}

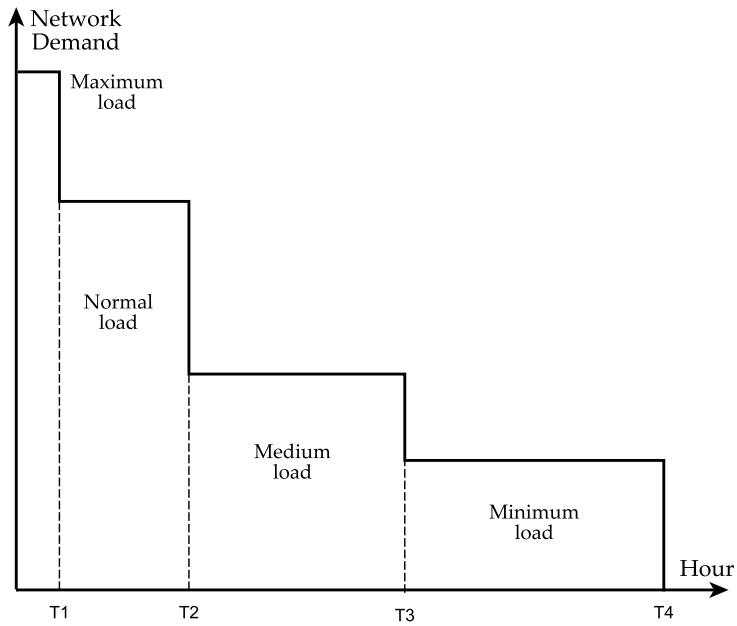

Figure 11: Load duration curve (adopted from [21])

\section{Extensions}

In this section we illustrate how the proposed GCAN procedure can be easily extended to take into account some other practical aspects related to the generation connection computations. Two aspects are considered: the inclusion of annual system losses [21, 22] in the procedure and the investigation of a possibility to use network reconfiguration along generation increase computations.

\subsection{Annual losses and network reconfiguration considerations}

As of annual losses, in order to preserve the pragmatism of the procedure, we suggest the use of a load duration curve discretized in four load bands (see Fig 11) [21]. The annual losses are estimated by the weighted average active power losses of the four bands,

$$
\begin{aligned}
P_{L a v}=\frac{1}{T_{4}} * & \left(T_{1} P_{L 1}+\left(T_{2}-T_{1}\right) P_{L 2}\right. \\
& \left.+\left(T_{3}-T_{2}\right) P_{L 3}+\left(T_{4}-T_{3}\right) P_{L 4}\right) .
\end{aligned}
$$




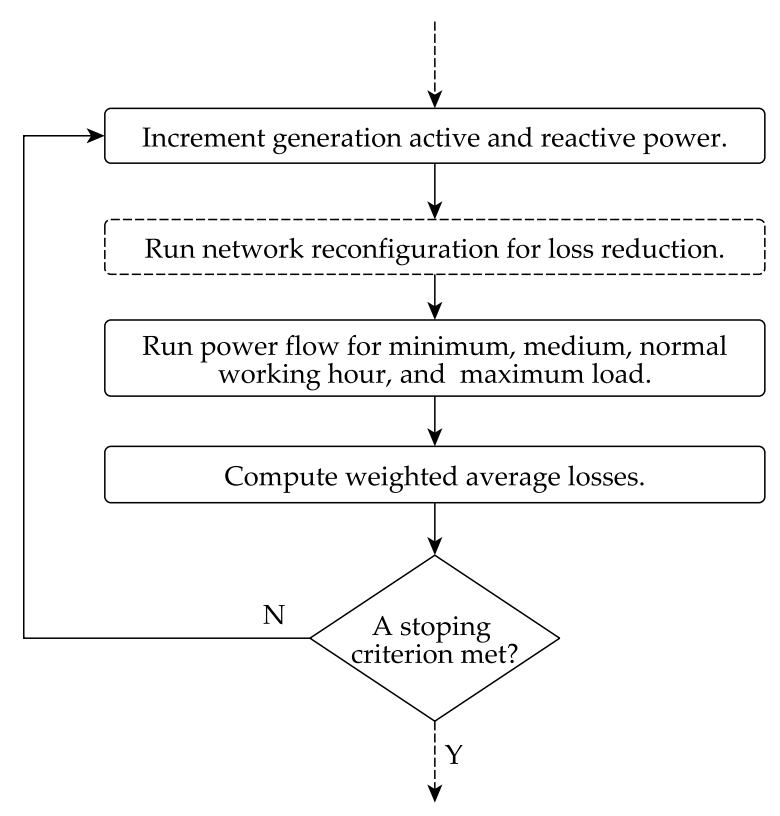

Figure 12: GCAN procedure with the target on weighted average annual losses and network reconfiguration run at each generation increase step.

where $P_{L 1}, P_{L 2}, P_{L 3}$, and $P_{L 4}$ represent the losses for maximal, normal working hour, medium, and minimum load conditions, respectively. Then the target value is set for these losses thus minimally changing the procedure presented in previous sections. The modified part of the procedure is shown in Fig 12 In the same figure the network reconfiguration run at each generation increase step is shown as an option. Since the proposed procedure is based on the use of existing tools, a network reconfiguration for loss reduction is considered.

The results obtained using the IEEE 33-bus test system are listed in Table 6. The same value for target losses used in the previous section for a system snapshot is set for weighted average losses while values related to load duration curve are set to: $T_{1}=73, T_{2}=2920, T_{3}=5840$, and $T_{4}=8760$. What is termed "reconfig-1" corresponds to the optimal network configuration computed once for the situation at the start of the GCAN procedure, and kept fixed through the procedure. The term "reconfig-2" denotes the case where network reconfiguration for loss reduction is run at each step of generation increase. All other simulation parameters are kept the same as in previous sections. The results reveal that solving network reconfiguration for loss reduction along GCAN computations ("reconfig2 ") increases the total connection power by $0.61 \mathrm{MW}$ and there is a slight difference in locating generations for two situations (the results differ in four locations where smaller generations are located: substation $11,29,30$, and 31 , while there is a good agreement on other substations). Network configurations in terms of open lines are given in Table 7 For "reconfig1 " network configuration is obtained by running the reconfiguration problem for loss reduction once and keeping it fixed through GCAN computations. The results for "reconfig-2" cor-
Table 6: GCAN results considering annual losses

\begin{tabular}{ccc}
\hline Substation & $\begin{array}{c}\text { MW } \\
\text { reconfig-1 }\end{array}$ & $\begin{array}{c}\text { MW } \\
\text { reconfig-2 }\end{array}$ \\
\hline 2 & 3.85 & 3.80 \\
\hline 3 & 3.14 & 3.10 \\
\hline 4 & 2.17 & 1.96 \\
\hline 11 & 0.00 & 0.57 \\
\hline 18 & 2.79 & 2.74 \\
\hline 19 & 0.68 & 0.66 \\
\hline 20 & 0.90 & 0.87 \\
\hline 22 & 1.48 & 1.46 \\
\hline 23 & 0.63 & 0.62 \\
\hline 24 & 0.33 & 0.32 \\
\hline 28 & 0.59 & 0.80 \\
\hline 29 & 0.67 & 0.00 \\
\hline 30 & 0.00 & 0.52 \\
\hline 31 & 0.00 & 0.52 \\
\hline Total & 17.23 & 17.94 \\
\hline & &
\end{tabular}

Table 7: Network configurations

\begin{tabular}{cc}
\hline Configuration & $\begin{array}{c}\text { Open } \\
\text { switches }\end{array}$ \\
\hline reconfig-1 & $6-7,8-9,13-14,24-28,31-32$ \\
\hline reconfig-2 & $4-5,6-7,8-9,12-13,30-31$ \\
\hline
\end{tabular}

respond to the final configuration when then GCAN procedure is stopped.

\subsection{Computational burden}

The computational burden of the GCAN procedure is dictated by the time needed for running power flow and network reconfiguration. For the IEEE 33-bus test system a power flow run takes in average 0.012 seconds while network reconfiguration needs in average 1.51 seconds (on Windows 7, Intel CORE i5, $2.30 \mathrm{GHz}$, 4GB RAM machine, using Matlab environment(32-bit version on 64-bit machine)). For both conditions ("reconfig-1" and "reconfig-2") 21 steps were needed to complete the GCAN procedure and the overall computation time is given in Table 8 . The computational burden is indeed much bigger if a reconfiguration problem for loss reduction is solved along GCAN computations. Computation time is increased with the RL system (which is a considerably larger, realistically sized system) and one power flow run takes in average 0.082 seconds while network reconfiguration takes 134.23 seconds in average. For this system 17 steps were needed to complete the GCAN computation, which gives overall times of 139.806 seconds for "reconfig-1" and 2287.486 seconds for

Table 8: Computational burden

\begin{tabular}{cc}
\hline Configuration & $\begin{array}{c}\text { Computation time } \\
\text { (seconds) }\end{array}$ \\
\hline reconfig-1 & 2.518 \\
\hline reconfig-2 & 34.228 \\
\hline
\end{tabular}




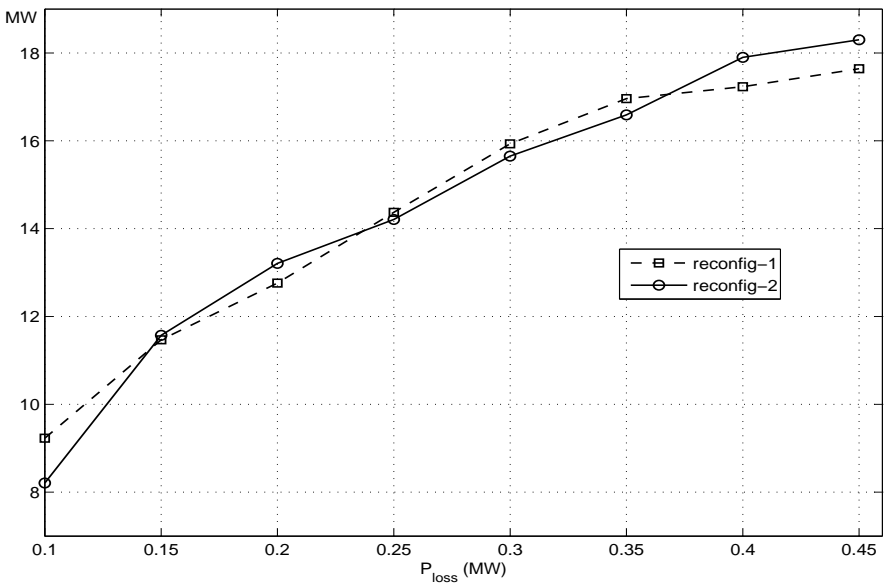

Figure 13: Generation connections as function of target losses

"reconfig-2". All computational times were obtained by employing good practice of repeated power flow computations:

- the power flow solution of each step is taken as the initial solution for the next one (for all four load bands),

- within the network reconfiguration problem the power flow solution for the first configuration is used as the initial one for all line tripping (in other words, the first solution is for all switches considered closed and used for every line trip in the first step as the initial solution, then the power flow solution with the best line definitively tripped is used as the initial one for all line tripping of that stage, and so on),

- the list of switches in network reconfiguration is formed and updated at each computation so that the ones leading to disconnected system are not considered, thus avoiding unnecessary computations [15].

Although the results of Table 6 suggest the network reconfiguration for loss reduction yields larger total connection amount, we further studied this problem for different targets set on weighted average losses. Figure 13 displays the results, for the IEEE 33-bus test system, showing the network reconfiguration for loss reduction, if ran at each generation increase step, does not always offer bigger connection amounts (on the contrary it sometimes slightly decreases them). This is not surprising since reconfiguration for loss reduction impacts the system voltage profile and thus possibly decreased the amount of connection when voltage magnitude upper limit is constraining (rural distribution systems), as in the cases considered in this paper.

Based on these observations and the computational time requirements, observed for IEEE 33-bus and RL system, we suggest an option with fixed configuration as more practical since simpler and faster, while its accuracy is not considerably compromised.

The results of GCAN computation for the RL system, for the first year, considering the target on weighted average annual losses with the same conditions as in previous related results are given in Fig 14. For this system locations of generation are

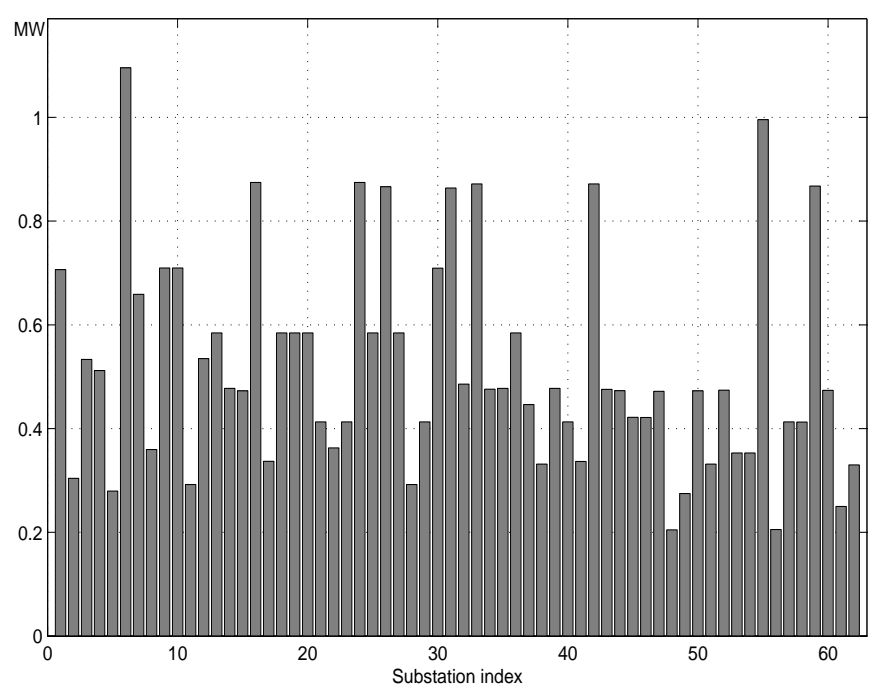

Figure 14: Generation connections for RL system with annual losses consideration: corresponds to the system one line diagram in Fig 7 (from left to right)

the same as obtained with the target on snapshot losses while amounts are different and the total capacity computed is 32.02 MW.

\subsection{Impact of the pre-defined minimum generation connection value}

The pre-defined minimum generation connection values used in the computations for IEEE 33-bus and RL systems were chosen by trying different values in the range $[0.1,1.0] \mathrm{MW}$. The choice of these threshold values impacts the results of the GCAN computation. Figure 15 shows the total connection amount for several values of pre-defined minimum generation connection. Smaller pre-defined minimum generation connection values yield a smaller total connection amount. Moreover choosing too large values also degrades the total connection amount (stopping criterion 3 of the RPF procedure is met). As a recommendation the choice of the threshold should be based on trying different pre-defined minimum generation connection values: in practice a DSO has rough guess of this value and should try several values around that guess.

\section{Discussion and future work}

Although the procedure is developed with reference to the European regulatory framework we believe it is applicable in cases where the DSO is allowed to own generation provided that the procedure is transparent. Investigation of possible adjustment for this situation constitutes a part of our future research efforts.

We found that a simple approximation of the distance from security boundary defined by equation (5) is a good compromise between computational burden of RPF and accuracy. Clearly, it is interesting how some other notions of distance could further improve performances of the proposed procedure. This also constitutes a part of our future research activities. 


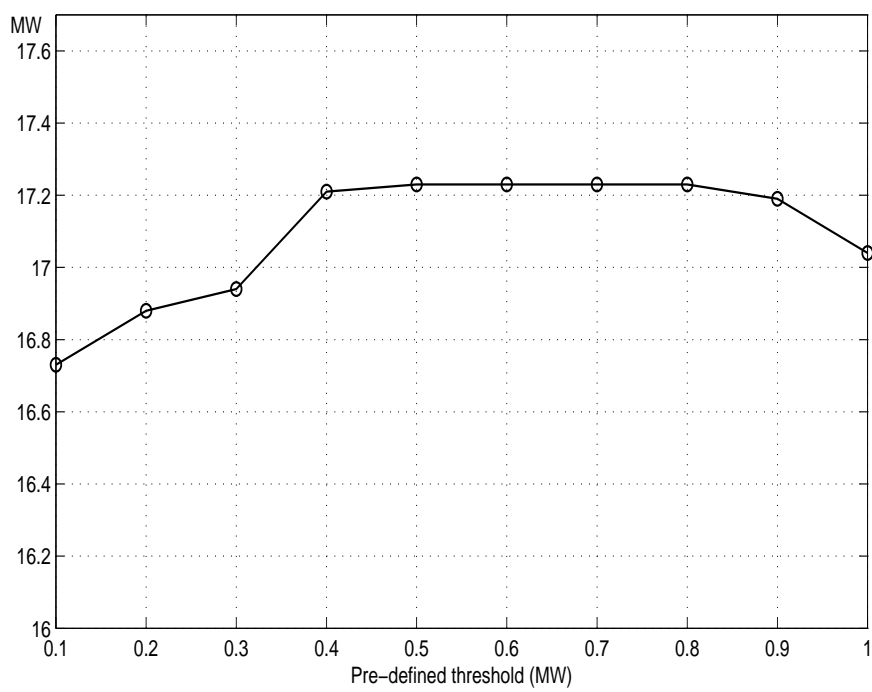

Figure 15: Total connection amount as a function of the pre-defined minimum generation connection value.

A comparison of the OPF and the RPF methods should not only be based on the optimality gap. First, the solver used to solve the MINLP of the OPF formulation has no guarantee to be globally optimal, hence there may exist a significantly better solution. Second, the GCAN analysis gives indications about good locations from the network's perspective, and a detailed study would be required for any connection request. In this respect, both approaches identify almost the same sets of buses, and differ only in the capacity. Finally, the RPF method, besides its simplicity, provides an intuitive way of solving this problem and additional information because it allows plotting figures such as Fig 8 , and thus gaining confidence on the overall solution obtained.

By performing a sensitivity analysis with the RPF and OPF methods, it is easy to observe that several substations are actually equivalent, but the solution algorithm eventually selects one of them. An alternative way to display the results of the GCAN computation could be to run a type of sensitivity analysis and indicate that several buses are equivalent from the DSO perspective, they can thus host approximately the same capacity, whereas some buses offer a large potential. Not considering the latter buses would significantly reduce the hosting capacity of the network. Displaying the information in this way may thus also lift any suspicion of a GCAN that would be very sensitive to the initialization of the algorithm. Our procedure computes firm generation, but providing indications about the maximum allowable generation achievable in a substation, but subject to some flexibility, is an important topic to further investigate.

The suggestion about the use of network reconfiguration holds true only for its variant to minimize system losses. It is certainly a challenge to devise an appropriate network reconfiguration with carefully chosen objective to be used within the proposed procedure. We presently investigate this possibility.

Related to the choice of technological solutions (synchronous, wind, PV), in this work it is assumed that DSO collects the information and knows preferred technological solu- tion for each candidate substation (based on prevailing weather conditions, land use issues to build specific generation, etc.) and we believe this is realistic in most of DSO systems. Consideration of different technological solutions even in a limited number of substations would dramatically increase computations burden of the proposed procedure and complicate publication of its results. If it happens that a preferred technological solution in a limited number of substations is not known or obvious, we suggest the DSO performs GCAN computations assuming a unity power factor for these substations.

\section{Conclusion}

A pragmatic procedure for global capacity announcement of distribution systems conducted by a DSO is proposed in this work. This procedure is not meant to be a replacement for more detailed computations for generation connection projects. Its purpose is to serve as the first step in the generation connection decision process and estimate the values of available connections capacity and thus encourage connection developers to start with the project. The proposed procedure relies on a power flow tool routinely used in DSOs practice and minimum requirements for scripting. An appropriate network reconfiguration algorithm is chosen (the one relying on a power flow tool) and a specific implementation of RPF is proposed. This implementation is able to solve one of the key problems in generation connections computation, namely the peril of network sterilization.

All the procedure is implemented in a rolling horizon manner, refreshed at each step of the planning horizon. The generation capacities are computed for a future system situation and projected back to the present situation.

Results using three test systems clearly show the potential of the proposed procedure to be used as a practical approach for approximate capacity computations followed by more detailed and rigorous steps of generation connection process.

\section{Acknowledgment}

This research is supported by Public Service of Wallonia, Department of Energy and Sustainable Building, within the framework of the GREDOR project.

\section{References}

\section{References}

[1] T. Lindl, K. Fox, A. Ellis, R. Broderick, Integrated distribution planning concept paper: A proactive approach for accommodating high penetrations of distributed generation resources, Tech. rep., IREC - Interstate Renewable Energy Council, available online: www.irecusa.org (2013).

[2] P. S. Georgilakis, N. D. Hatzargyriou, Optimal distributed generation placement in power distribution networks: Models, methods, and future research, IEEE Trans. Power Syst. 28 (2013) 3420-3428.

[3] A. Keane, M. O'Malley, Optimal allocation of embedded generation on distribution networks, IEEE Trans. Power Syst. 20 (2005) 1640-1646.

[4] Directive 2005/89/ec of the european parliament and of the council of 18 january 2006 concerning measures to safeguard security of electricity supply and infrastructure investment, Tech. rep., European Parliament (2005). 
[5] Distributed generation ownership issues, review of current practices, future options and european policy recommendation, Tech. rep., European Commission, available online: ec.europa.eu (2007).

[6] R. Cossent, T. Gomez, P. Frias, Towards a future with large penetration of distributed generation: Is the current regulation of electricity distribution ready? regulatory recommendations under a european perspective, Energy Policy 37 (2009) 1145-1155.

[7] M. da Graca Carvalho, Eu energy and climate change strategy, Energy 40 (2012) 19-22.

[8] P. Siano, L. F. Ochoa, G. P. Harrison, A. Picollo, Assessing the strategic benefits of distributed generation ownership for dnos, IET Gener., Trans., Dist. 3 (2009) 225-236.

[9] Décret du 11 avril 2014 modifiant le décret du 12 avril 2001 relatif á l'organisation du marché régional de l'électricité et modifiant le décret du 19 décembre 2002 relatif á l'organisation du marché régional du gaz, Tech. rep., Parlement Wallon, Namur, Belgium (2014).

[10] G. P. Harrison, A. R. Wallace, Optimal power flow evaluation of distribution network capacity for the connection of distributed generation, IEE Proc., Gener., Trans., Dist. 152 (2005) 115-122.

[11] H. M. Ayres, W. Freitas, M. C. D. Almeida, L. C. P. D. Silva, Method for determining the maximum allowable penetration level of distributed generation without steady-state voltage violations, IET Gener., Trans., Dist. 4 (2010) 495-508.

[12] Station and feeder capacity calculator, Tech. rep., HydroOne, Canada, available online: www.hydroone.com (2015).

[13] Long term development statement, Tech. rep., Western Power Distribution, available online: www.westernpower.co.uk (2015).

[14] D. Ernst, The GREDOR project: Gestion des réseaux electriques de distribution ouverts aux renouvelables, Tech. rep., University of Lieège, available online: www.gredor.be (2013).

[15] F. V. Gomes, S. Carneiro, J. L. R. Pereira, M. P. Vinagre, P. A. N. Garcia, L. R. Araujo, A new heuristic reconfiguration algorithm for large distribution systems, IEEE Trans. Power Syst. 20 (2005) 1373-1378.

[16] F. Capitanescu, L. F. Ochoa, H. Margossian, N. D. Hatzargyriou, Assessing the potential of network reconfiguration to improve distributed generation hosting capacity in active distribution systems, IEEE Trans. Power Syst. 1 (2015) 346-356.

[17] Y. Ou, C. Singh, Assessment of available tranfer capability and margins, IEEE Trans. Power Syst. 17 (2002) 463-468.

[18] R. D. Zimmerman, C. E. Murillo-Sanchez, R. J. Thomas, Matpower: Steady-state operations, planning and analysis tools for power systems research and education, IEEE Trans. Power Syst. 26 (2011) 12-19.

[19] W. E. Hart, C. Laird, J.-P. Watson, D. L. Woodruff, Pyomo-optimization modeling in python, Vol. 67, Springer Science \& Business Media, 2012.

[20] P. Bonami, J. Lee, BONMIN users manual., Tech. rep. (2006).

[21] G. P. Harrison, A. Piccolo, P. Siano, A. R. Wallace, Exploring the tradeoffs between incentives for distributed generation developers and dnos, IEEE Trans. Power Syst. 22 (2007) 821-828.

[22] L. F. Ochoa, G. P. Harrison, Minimizing energy losses: Optimal accommodation and smart operation of renewable distributed generation, IEEE Trans. Power Syst. 26 (2011) 198-205.

[23] J. F. Dopazo, O. A. Klitin, A. G. W. Stagg, M. Watson, An optimization technique for real and reactive power allocation, Proceedings of the IEEE 55 (1967) 1877-1885.

\section{Appendix}

Optimal power flow, used in section 4.3 for validation purposes, is formulated as [10],

$$
\max _{P_{g}} f\left(P_{g}\right)=\sum_{i \in C S L}-C_{i} P_{g_{i}}
$$

subject to:

$$
\begin{aligned}
h\left(P_{g}, x\right) & = \\
Q_{g_{i}} & =P_{g_{i}} \tan \theta_{i}, i \in C S L \\
V_{\text {min }_{j}} \leq V_{j} & \leq V_{\max _{j}}, j \in N \\
S_{k} & \leq S_{\text {max }_{k}}, k \in N_{l} \\
0.5 \leq & P_{g_{i}} \leq S C R_{i}, i \in C S L \\
P_{\text {loss }} \leq & 0.40
\end{aligned}
$$

where:

- $P_{g}$ the vector of active power generation,

- $Q_{g}$ the vector reactive power generation,

- $V$ the vector of bus voltage magnitude,

- $S$ the vector of apparent power in the line,

- $C$ the cost of active power associated with considered generator,

- $N$ the number of buses,

- $N_{l}$ the number of lines,

- $\theta$ the angle corresponding to power factor of generator,

- $x$ the vector of state variables (bus voltage magnitudes and angles),

- $h$ the vector of power flow equations,

- $P_{\text {loss }}$ total active power losses in the system.

Total active power losses are computed as [23],

$$
P_{\text {loss }}=\sum_{m=1}^{N} \sum_{n=1}^{N}\left(\alpha_{m n}\left(P_{m} P_{n}+Q_{m} Q_{n}\right)-\beta_{m n}\left(P_{m} Q_{n}-Q_{m} P_{n}\right)\right)
$$

with:

$$
\begin{aligned}
\alpha_{m n} & =\frac{R_{m n}}{V_{m} V_{n}} \cos \left(\phi_{m n}\right) \\
\beta_{m n} & =\frac{R_{m n}}{V_{m} V_{n}} \sin \left(\phi_{m n}\right)
\end{aligned}
$$

where:

- $P_{m}$ active power injection in bus $m$,

- $Q_{m}$ reactive power injection in bus $m$,

- $\phi_{m n}$ the difference of voltage phase angles in buses $m$ and $n$,

- $R_{m n}$ the real component of the bus impedance matrix at position $m, n$.

Note that line apparent powers are used as proxy for thermal line constraints. 